\title{
EDITORIAL AND COMMENT Use of PROs in Primary Care: PROMIS or Disappointment?
}

\author{
Elizabeth R. Pfoh, PhD, MPH and Michael B. Rothberg, MD, MPH \\ Center for Value-Based Care Research, Cleveland Clinic, Cleveland, OH, USA.
}

J Gen Intern Med 33(8):1207-8

DOI: $10.1007 / \mathrm{s} 11606-018-4511-\mathrm{x}$

(c) Society of General Internal Medicine 2018

$I^{n}$ n 2004, the National Institutes of Health (NIH) launched its Roadmap as a way to transform healthcare research by making it more efficient. ${ }^{1}$ PROMIS (the Patient-Reported Outcomes Measurement System) was developed as part of the Roadmap to fill the need for an evidenced-based, stateof-the-art assessment system for measuring self-reported health. ${ }^{1}$ PROMIS standardizes collection of patient-reported outcomes (PROs) (such as depression and anxiety) across research studies and enables efficient assessment of various aspects of self-reported health. Further, PROMIS provides a way to capture the patient's full experience of their disease and treatment. ${ }^{1}$ In the clinical setting, physicians and researchers can assess symptoms, inform treatment decisions, and track outcomes using PROs. ${ }^{2}$

SPADE symptoms (sleep problems, pain, anxiety, depression, and low energy/fatigue) are prevalent and can be measured using PROMIS. They are the types of outcomes the NIH was hoping to improve. In this month's Journal of General Internal Medicine, Kroenke and colleagues' report results of a randomized trial in which they offered primary care clinicians a visual display of patients' SPADE symptoms, then measured the change in 3-month composite SPADE scores. ${ }^{3}$ They found no difference in SPADE symptom improvement or clinician action between patients whose clinicians saw the visual displays and the control group. Documented clinician action included medication prescriptions (66\% of study participants) followed by other treatments such as education (36\%) or referrals (28\%). Patient-reported clinician action was not associated with patient characteristics nor satisfaction. Importantly, two thirds of threshold-level symptoms were unresolved at 3-month follow-up. ${ }^{3}$

While disappointing, the findings are unsurprising. The study's randomized clinical trial design provides strong evidence that measuring symptoms alone does not change practice. Displaying patients' symptom scores did not improve documentation, symptom-specific clinician action, or patient satisfaction. There are three potential explanations: (1) physicians overlooked the displays; (2) clinicians prioritized other diseases over SPADE symptoms; or (3) SPADE symptoms are

Published online June 5, 2018 difficult to treat. While interviewing the clinicians could answer these questions definitively, some insight can be gleaned from the clinicians' actions and interviews with the patients. The first explanation seems unlikely because according to the patients, the vast majority of threshold symptoms were discussed. ${ }^{3}$ Patients also reported that clinicians often prioritized other diseases. In fact, the most common reason for not discussing the SPADE symptom was a more pressing medical issue $(49 \%)$. Finally, many patients reported not needing (47\%) or wanting (29\%) treatment. Yet, treatment was desired the majority of the time when symptoms were threshold level for pain, sleep, and fatigue. ${ }^{3}$

Perhaps the most likely reason that presenting the information did not result in symptom improvement is that SPADE symptoms are difficult to treat. Until recently, opioids were the most common treatment for pain. Opioids are addictive and increasingly regulated. There are medications for sleep, anxiety, and depression, too, but their effectiveness is low and side effects are common. Cognitive behavioral therapy (CBT) is effective but patients may face barriers to accessing an expert in CBT, including insurance, time, or availability. As the authors acknowledge, the lack of systematic supports to address patients' symptoms likely blunted the impact of the information provided. ${ }^{3}$ Prior randomized trials of depression screening found screening improved morbidity and mortality only when substantial supports are in place. ${ }^{4}$ Better treatments are clearly needed.

If we cannot readily improve SPADE symptoms, does it still make sense to measure them? Historically, patients have shared their medical history and symptoms for their own benefit, for the purposes of diagnosis and treatment. Routine measurement can improve outcomes for patients with chronic disease (e.g., monitoring blood pressure or arthritis symptoms), but this assumes we have effective treatments available. Monitoring SPADE symptoms may be more akin to monitoring body mass index, an often futile and frustrating activity for physician and patient alike. Yet, to improve overall healthcare quality, learning health systems need data, especially the kind provided by patients completing PROMIS measures. Learning health systems can identify ways to improve care delivery by leveraging these data using real-time analysis. ${ }^{5}$ Researchers can link PROs and electronic health record data to understand the effectiveness of the treatments they provide. They can study positive deviants - physicians whose patients improve more than the average - to spread their practices. A defined set of measures for SPADE symptoms, such as one used in this 
study, can provide a basis for health systems to compare themselves to others and assess the effectiveness of different systematic supports. This goes beyond simply measuring symptoms. We must identify the interventions (i.e., pharmacological, cognitive behavioral therapy, or otherwise) that improve patients' symptoms in the real world and subject these interventions to rigorous evaluation. Of course, gathering and analyzing these data in the course of a busy practice is not simple, ${ }^{6}$ neither is treating insomnia, pain, anxiety, fatigue, and/or depression. Routinely measuring SPADE symptoms is a requisite step toward the latter.

Currently, some health systems ask patients to report a limited number of PROs to meet quality measures. For example, depression screening using a PRO is an accountable care organization quality measure tied to payment. ${ }^{7}$ However, as more PROs are integrated into primary care, the burden on patients will increase. Health systems will likely ask patients to share more about their health at more routine intervals. However, unlike a review of systems, or screening questions about depression or anxiety, the PROMIS is unlikely to be used directly in the patient's care. Patients, then, may rightly resist sharing their information.

One way to get patients to provide their information could be to appeal to their altruism. Another is to allow them to track their progress. The case of Amazon illustrates that providing data can be supply an individual and a public good. One of Amazon's earliest innovations was allowing customers to post book reviews, which were positively associated with book sales. $^{8,9}$ Individuals who posted reviews found satisfaction in recording their impression of the book, ${ }^{10}$ and the public benefited from access to unbiased reviews. Of note, the vast majority of Amazon customers never post a review. In research, if only a tiny fraction of patients routinely complete PROMIS measures, conclusions based on the data may be biased. Thus, engagement in sharing health information needs to be high. This is particularly true as health systems compete with almost every other industry to obtain patient's opinions, experiences, and data. In this domain, healthcare organizations may have an edge over other businesses, however, because patients desire to help other patients is likely greater than their desire to help movie-goers pick a film or vacationers choose an Airbnb. In addition, patients may appreciate the ability to track their symptoms over time and compare themselves to national norms. Ultimately, healthcare systems must demonstrate to patients that sharing this information is worthwhile, or identify a way to compensate patients for their time.
To demonstrate value to patients, learning health systems should invest in measuring PROs, enable access to appropriate interventions for patients, and continuously improve patient care. Kroenke and colleagues' study demonstrates that SPADE symptoms can be systematically measured in the primary care setting. ${ }^{3}$ The vital next step is using this information to improve population health. Let us hope that patients understand that while reporting their symptoms may not improve their individual health today, it may help to improve the health of patients like them tomorrow.

Corresponding Author: Elizabeth R. Pfoh, PhD, MPH; Center for Value-Based Care Medicine Cleveland Clinic, 9500 Euclid Avenue, G10, Cleveland, OH 44195, USA (e-mail: Pfohe@ccf.org).

\section{Compliance with Ethical Standards:}

Conflict of Interest: The authors disclose no conflicts of interest.

\section{REFERENCES}

1. A Decade of Discovery: The NIH Roadmap and Common Fund. National Institutes of Health, 2014. Available at https://commonfund.nih.gov/ sites/default/files/ADecadeofDiscoveryNIHRoadmapCF.pdf.) Accessed April 10, 2018

2. Lavallee DC, Chenok KE, Love RM, et al. Incorporating PatientReported Outcomes Into Health Care To Engage Patients And Enhance Care. Health Aff (Millwood) 2016;35:575-82.

3. Kroenke K, Talib TL, Stump TE, Kean J, Haggstrom DA, DeChant $\mathbf{P}$, Lake KR, Stout M, Monahan PO. Incorporating PROMIS Symptom Measures into Primary Care Practice: A Randomized Clinical Trial. Journal of General Internal Medicine. 2018 (SPI 4391)

4. O'Connor EA, Whitlock EP, Gaynes B, Beil TL. Screening for Depression in Adults and Older Adults in Primary Care: An Updated Systematic Review. Rockville (MD)2009.

5. Bernstein JA, Friedman C, Jacobson P, Rubin JC. Ensuring public health's future in a national-scale learning health system. Am J Prev Med 2015;48:480-7.

6. Bindman AB, Pronovost PJ, Asch DA. Funding Innovation in a Learning Health Care System. JAMA 2018;319:119-20.

7. Centers for M, Medicaid Services HHS. Medicare Program; CY 2018 Updates to the Quality Payment Program; and Quality Payment Program: Extreme and Uncontrollable Circumstance Policy for the Transition Year. Final rule with comment period and interim final rule with comment period. Fed Regist 2017;82:53568-4229.

8. Amazon-how one controversial decision ignited massive growth. Available at: https://digit.hbs.org/submission/amazon-how-one-controversial-decision-ignited-massive-growth. Accessed April 4, 2018.

9. Chevalier JA, Mayzlin D. The Effect of Word of Mouth on Sales: Online Book Reviews. Journal of Marketing Research 2006;43: 345-354

10. Levitt SD. Why do people post reviews on Amazon? 2005 Available at: http://freakonomics.com/2005/07/22/why-do-people-post-reviews-onamazon/?c_page=1\#comments_archived. Accessed April 10, 2018 